\title{
Répartition de la sève brute entre les jeunes rejets de bouleau étudiée à l'aide d'un phytocide
}

\author{
M. BEDENEAU a L. PAGES \\ I.N.R.A., Station de Recherches sur la Forêt et l'Envirommement \\ Centre de Recherches d'Orléans \\ Ardon, F 45160 Olivet
}

\begin{abstract}
Résumé
L'étude de lat répartition de la sève brute depuis les racines jusqu'aux différents rejets de cépées est ici réaliséc grâce au marquage de la sève ascendante par un phytocide. Sur 15 cépées d'un taillis de bouleau de 3 ans, des injections de phytocide dans les racines et lobservation des dégâts sur les rejets ont permis de constater que les souches ne peuvent être séparées en secteurs indépendants.
\end{abstract}

\section{Introduction, origine des rejets}

Le bouleau, comme beaucoup d'autres feuillus, donne naissance à de nombreux rejets après coupe rase du pied-mère. Ces bourgeons naissent dans la région du collet ou à la base des racines. D'après Fink (1980), qui a observé les bourgeons de Betula verracosa. les bourgeons proventifs donnent plutôt naissance à des racines.

Juste après la coupe, les rejets sont nombreux et regroupés en boutons épicormiques comme sur leucalyptus (RIEDACKER, 1973). Quand le taillis vieillit, le nombre de rejets et de boutons diminue. Vingt-cing ans après le recépage, il ne reste plus qui l à 5 rejets par cépéc dans les taillis classiques de bouleau.

\section{Objectifs}

Il s'agit d’analyser les relations qui existent entre les différents rejets de la cépée. Les brins peuvent êre alimentés de façon plus ou moins autonome par les racines et c'est ce degré d'autonomie que nous voulons apprécier. Une racine donnée alimente-t-elle indifféremment n'importe quel brin ou bien n'est-elle en relation qu’avec une zone restreinte de la cépée? Est-il possible de séparer la souche en secteurs indépendants? 

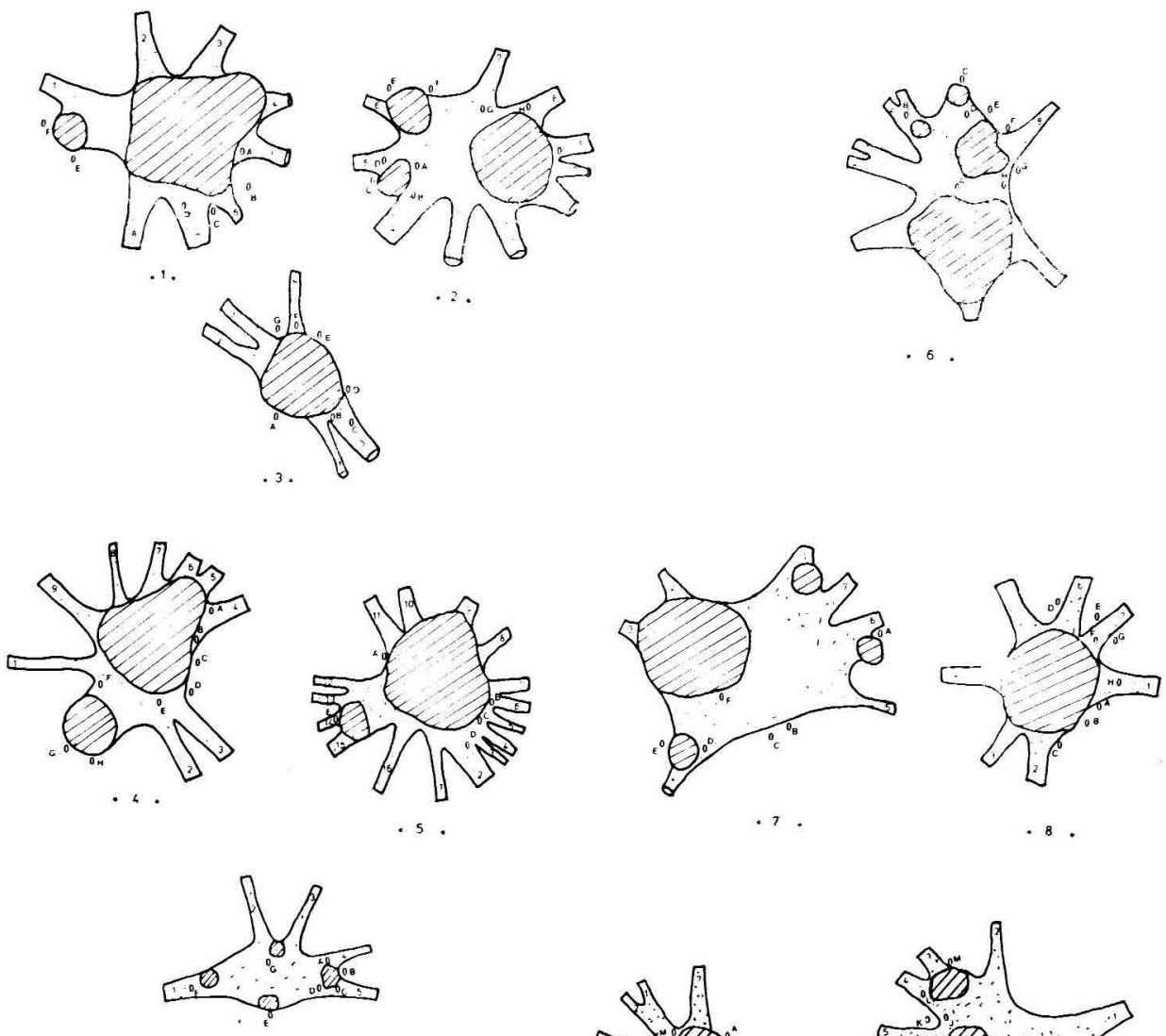

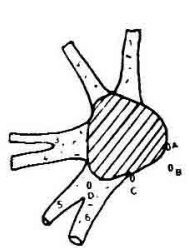

. 10
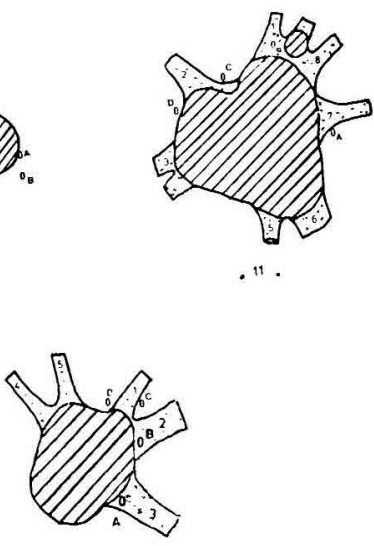
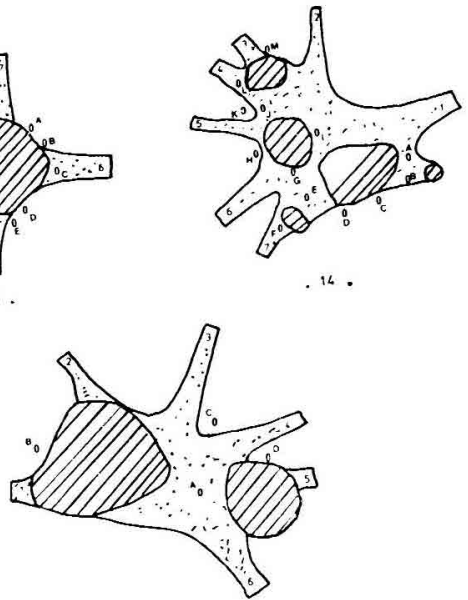

.15

Fici. 1 
Sur leucalyptus, RIEDACKer (1973) a montré que la souche pouvait être découpée en 4 secteurs indépendants lors de sa deuxième révolution, lat sectorisation disparaissant ensuite. Sur le chêne-liège, Destremeau \& Rodrrbourg (1969) ont observé lindépendance trophique des brins d’une même cépée. I.assoudtere (1980) a mis en évidence de fortes relations entre les rejets de bananier au second cycle.

L'étude qui suit concerne le bouleau. Il s’agit de premiers résultals, obtenus avec une technique nouvelle: il serait imprudent, pour l'instant. d'en tirer de véritables conclusions au niveau des translerts.

\section{Matériel et méthode}

Nous ávons expérimenté sur un taillis de bouleału (hybride présumé entre betula verracosa et Betula pubescens) dont la partie aérienne avait 3 ans. situé en Sologne. atix coordonnées 596-281 Lambert II, zone centrale.

Les é́pées ont subi un nombre variable de révolutions. Le diamètre de base des souches varie entre 30 et $80 \mathrm{~cm}$. Au moment de l'expérience, la hauteur maximale des brins était de $1,20 \mathrm{~m}$. leur diamètré à lá base varriait entre $0.5 \mathrm{ct} 2 \mathrm{~cm}$.

Le produit injecté est un mélange de phythormones $(2 / 3$ de $2-4-1$ ) et $1 / 3$ de 2-4-jT à $600 \mathrm{~g} / 1)$ utilisé comme débroussaillant systémique et vendu sous la référence Débroussaillant-600, Rhodiagri. Ce produit diffuse bien, il entraine une torsion des pousses et un dessèchement des feuilles. Un $\mathrm{cm}^{3}$ de ce produit fut injecté dans chaque racine traitée (cette quantité fut estimée à partir des doses généralement appliquées sur les trones, en se référant à la taille des bases des brins de la révolution précédente).

la couche de sol superficielle recouvrant les acines latérales a été enlevée sur un rayon de $50 \mathrm{~cm}$ autour de chaque cépée échantillon. Cette cépée fut ensuite dessinée pour montrè sá physionomie : chicots de la révolution précédente, position des boutons épicormiques, départs des racines mâttresses (cl. fig. 1).

L'injection fut ensuite réalisée avec une seringue de $1 \mathrm{~cm}^{3}$. après lorage d'un trou de $1 \mathrm{~cm}$ de diamètre sur $1 \mathrm{~cm}$ de profondeur, à environ $40 \mathrm{~cm}$ du centre de la cépée.

FIG. I

Physionomie des cépeseschantillons, numérotées de $/$ à $t 5$.

Plysionomy of the somple stumps, numbered from $t$ to $/ 5$.

- Les parties hachurées représentent les bases des brins de la révolution préeédente.

- Les boutons épicormiques sont repérés par des lettres.

- Les départs des racines maîtresses sont repérés par des numéros.

- The hatched areas show the remaining basis of the cat sprouts.

-. The groups of shoots are lettered.

- The starting point of main roots is mumbered. 


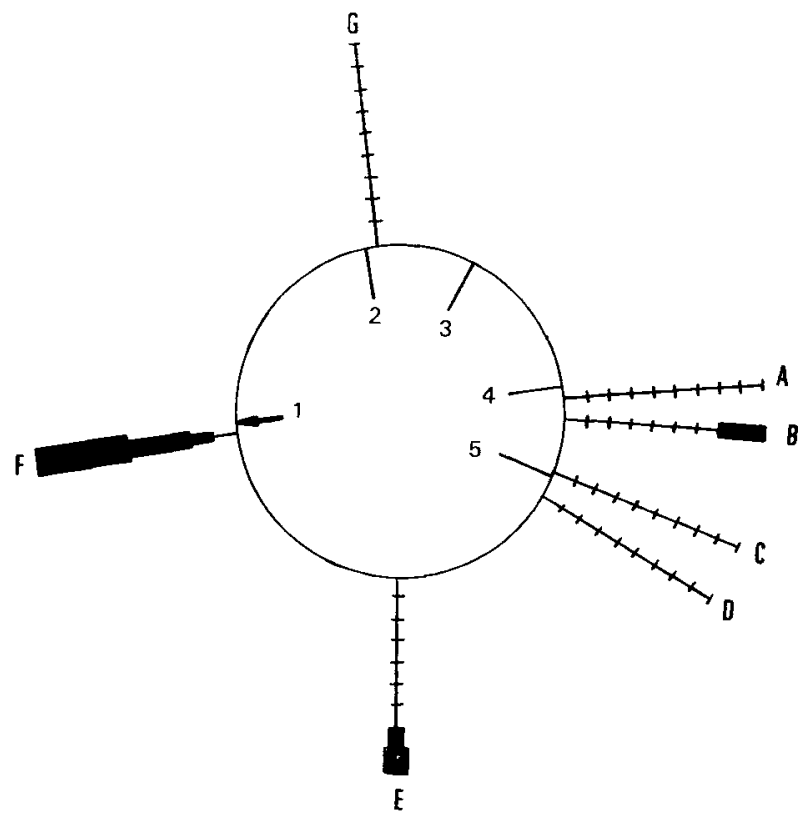

.9.

FIti. 2

Exemple de représentation des dégâts.

Example of representation of damage.

La cépéc est représentéc par un cercle. A l'intérieur de ce cercle, des segments de rayons numérotés (ici de $I$ à 5 ) montrent les racines-mères avec leur orientation (- si la racine n’a pas subi d'injection, - $\rightarrow$ si la racine a subi une injection). A l'extérieur du cercle, les segments de rayons indicés (ici de A a $(\mathrm{G})$ représentent les boutons avec leur orientation. Les dégâts subis par ces boutons sont symbolisés par de petits rectangles de largeur proportionnellc à la notation.

0 :

$1:$

2:

3 :

4 :

The stump is represented by a circle. Inside this circle, the mumbered radius segments (here from 1 to 5) show the main roots with their orientation (- if the root has not andergone an injection, $\rightarrow$ if the root has undergone an injection). Outside the circle, the lettered radius segments there from $A$ to $G$ ) show the gromps of shoots with their orientation. The damage andergone by the groups of shoots is symbolized by small rectangles, the width of which is proportional to the mark. 
Les cépées ont été choisies au hasard, éloignées les unes des autres d'au moins $6 \mathrm{~m}$ pour éviter les éventuelles interactions entre elles. Cinq traitements ont été retenus, et chacun d'eux appliqué sur 3 cépées :

1 - Le sol a été enlevé puis remis par-dessus les racines latérales ;

I! - Injection dans toutes les racines latérales ;

III - Injection dans une seule des racines latérales;

IV - Injection dans 2 racines latérales contiguës;

$\mathrm{V}$ - Injection dans 2 racines latérales séparćes par une troisième.

Toutes ces injections ont eu lieu le 14 avril 1982, une semaine environ après le débourrement.

L'observation des dégâts subis par la partic aérienne s'est faite par bouton épicormique (les dégâts se sont d'ailleurs montrés homogènes à l'intérieur du bouton). Nous avons donc regroupé les brins d'un même bouton, et avons attribué une note pour les dégâts subis par le feuillage des brins d'un bouton de la façon suivante :

- $0=$ aucun dégât ;

- 1 = quelques feuilles se déforment sans être décolorées : les dégâis sont jugés réversibles ;

- 2 = moins de la moitié du feuillage est décolorée : les dégâts sont jugés irréversibles ;

- 3 = plas de la moitié du feuillage est décolorée;

- 4 = toutes les feuilles soni décolorées.

Les premiers dégâts (déformation de feuilles, torsion de pousses) sont apparus le 3 mai 1982, après un radoucissement climatique. Les observations suivantes ont eu lieu tous les 3 jours jusqu'au $I^{\text {"r }}$ juin, puis toutes les semaines pendant ie mois de juin. L'évolution des dégâts s'est terminée fin juin. Les notations des dégâts ont été réalisées par le même obscrvateur durant toute la durée de l'expérience.

De façon à visualiser les cépées avec les dégâts qu'elles ont subis en fonction des injections, nous avons réalisé des schémas synthétiques (cf. fig. 2).

\section{Résultats}

4.1. Lorsquion enlève et rentet seulement le sol, sans injection. les parties aćriennes ne subissent aucun dégât, ce qui implique que les dégâts observés dans les autres traitements sont imputables à l'action du phytocide.

4.2. Lorsque toutes les racinés subissent une injection (cf. fig. 3), les dégâts sont très importants. On constate toutefois que certains boutons n'ont pas beaucoup réagi.

4.3. Lorsqu'une seule racine subil une injection (cf. fig. 4) :

Sur la cépée n" 7, 2 boutons (D et E) sur 6 n’ont subi aucun dégât. Ces 2 boutons sont situés lun à côté de l'autre et sont isolés sur une racine. De même, A, placé près d'une racine, a peu réagi. 

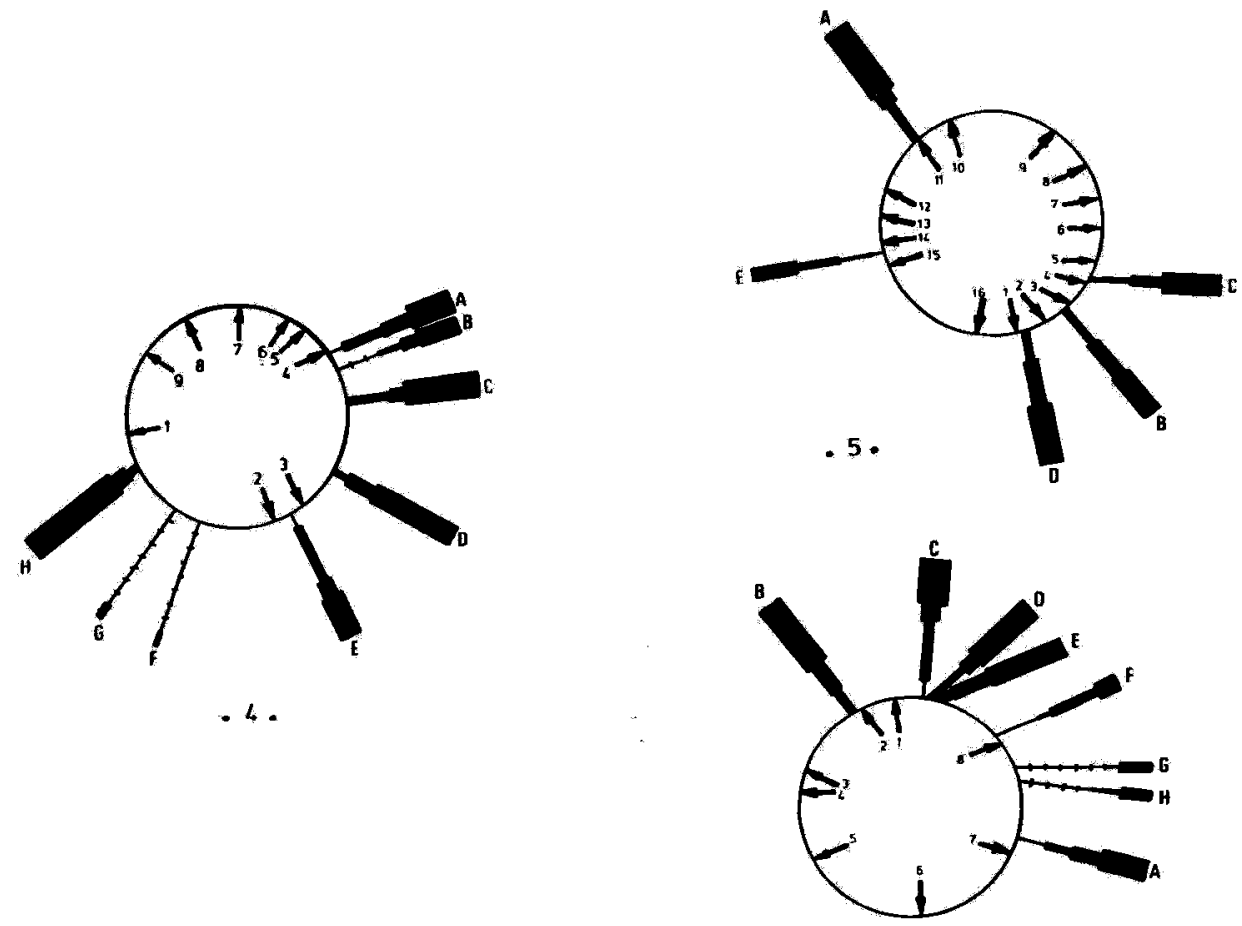

. 6 .

Fici, 3

Cépées ayant subi le traitement numéro 11 . répartition des dégâts.

Stumps which have andergone the treatment ntmber 11 . damage distribution. 

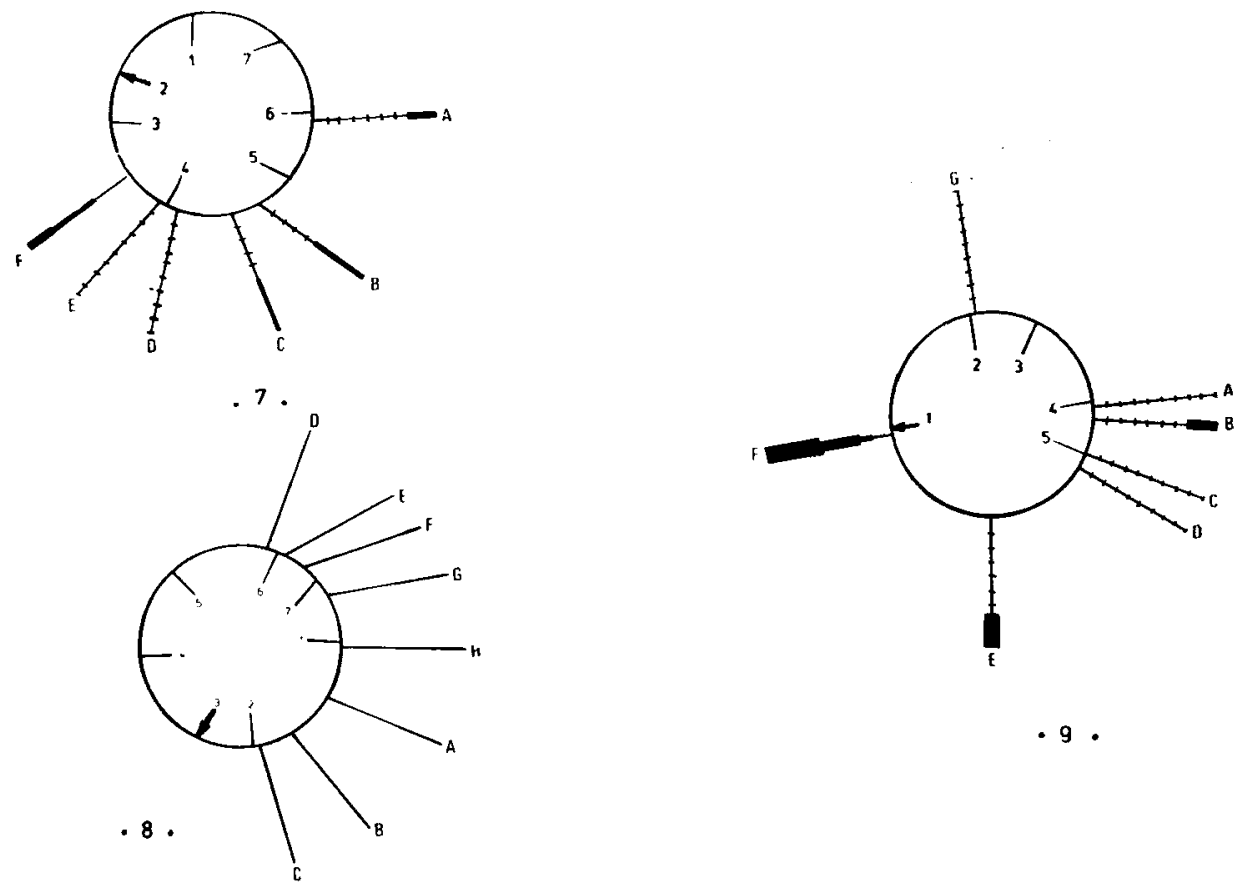

- 9 .

I'ICi. 4

Cépées ayamt subi le traitememt muntéro 11 , répartition des dégâts.

Stumps which have andergone the treatment number $I I$, damage distribution.

Sur la cépéc n" 8 , aucun bouton n'a été touché.

Sur la cépée $n^{\prime \prime} 9$, ce sont surtout les boutons $E$ et $F$, situés près de la racine traitée qui ont été touchés. Le bouton B, situé à l'opposé, présente lui aussi quelques dégâts. 
4.4. Lorsque 2 racines contiguës suhissent une injection (cf. fig. 5) :

Sur la cépée n"10, tous les boutons ont ressenti l'effet du traitement. Ce sont les boutons $\mathrm{A}$ et $\mathrm{B}$, les plus proches des racines traitées, qui ont subi le plus de dégâts. On remarque que les boutons $\mathrm{C}$ et $\mathrm{D}$ commencent à êlre atlaqués à la fin des observations.

Sur la cépée $n^{\prime \prime} 11$, ce sont seulement les boutons C et D. situés à proximité des racines traitées qui ont élć touchés, et de façon importante.

Sur la cépée $n^{\prime \prime} 12$, scul le bouton $A$, situé près d'une racine injectée, a subi de nets dégâts.

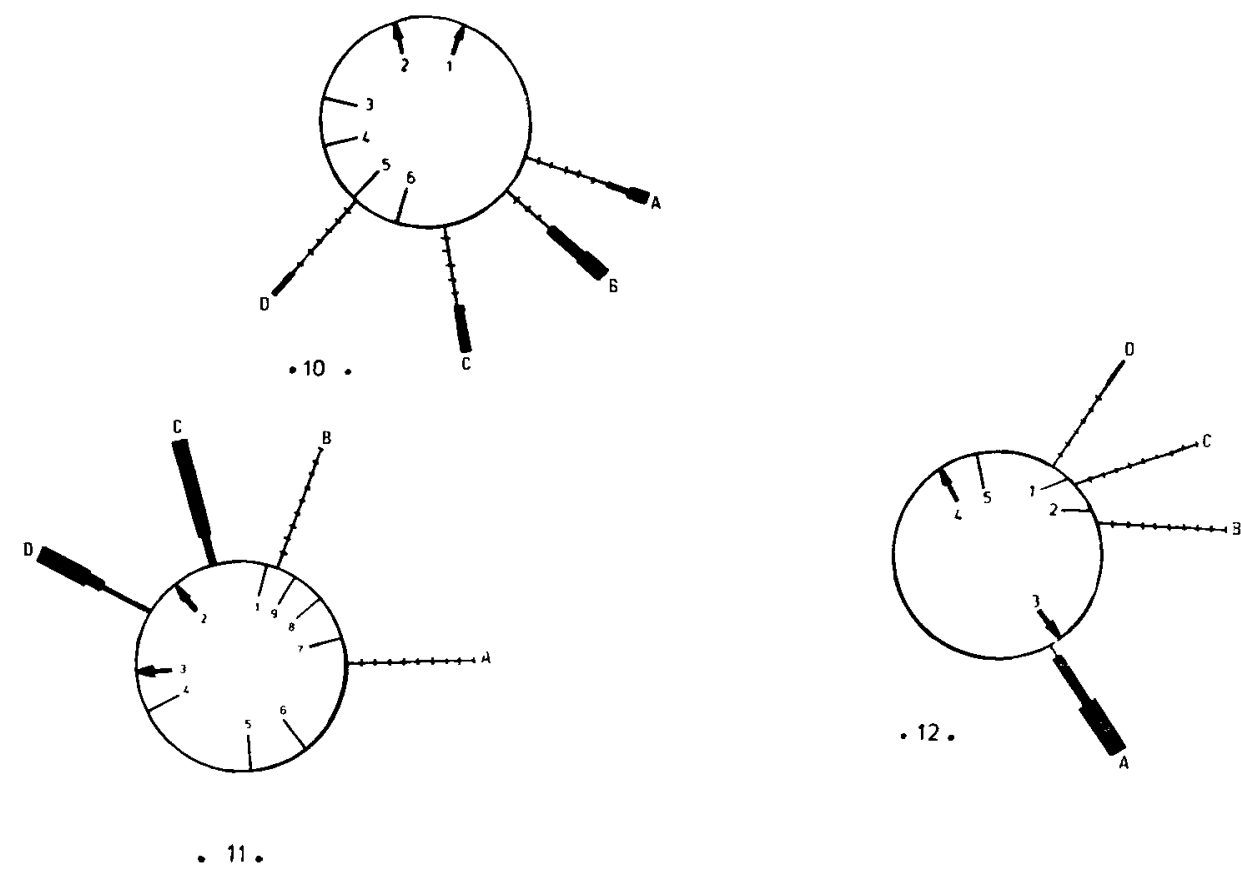

Fici. 5

Cépées ayant subi le traitement numéro $I V$. répartition des dégâts.

Stumps which have andergone the treatment number $I V$, damage distribution. 
4.5. Lorsque 2 racines séparées par une troisième subissent une injection (cf. fig. 6) :

Sur la cépée $n^{\circ} 13$, tous les boutons présentent des dégâts. La corrélation entre limportance de ceux-ci et la position par rapport à la racine traitée n'est pas évidente.

Sur la cépéc $n^{\prime} 14$, les boutons situés près de la racine $n^{\circ} 3$ ont nettement été touchés. En revanche, les boutons situés à proximité de la racine $\mathrm{n}^{\prime} 1$ ne présentent aucun dégât.

Sur la cépée no 15 , on observe peu de corrélation géographique entre la position du bouton par rapport aux racines traitées et l'importance des dégâts qu'il a subis.

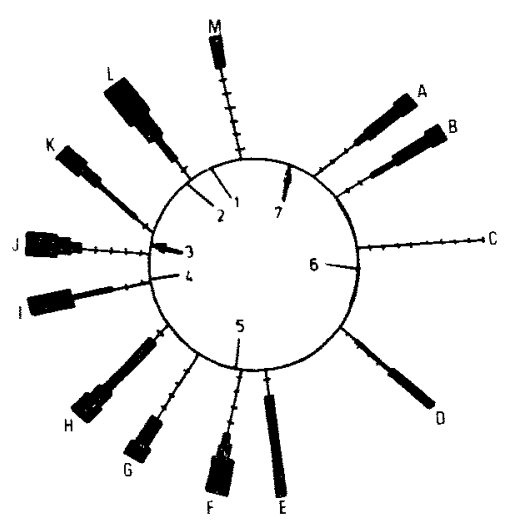

. 13.
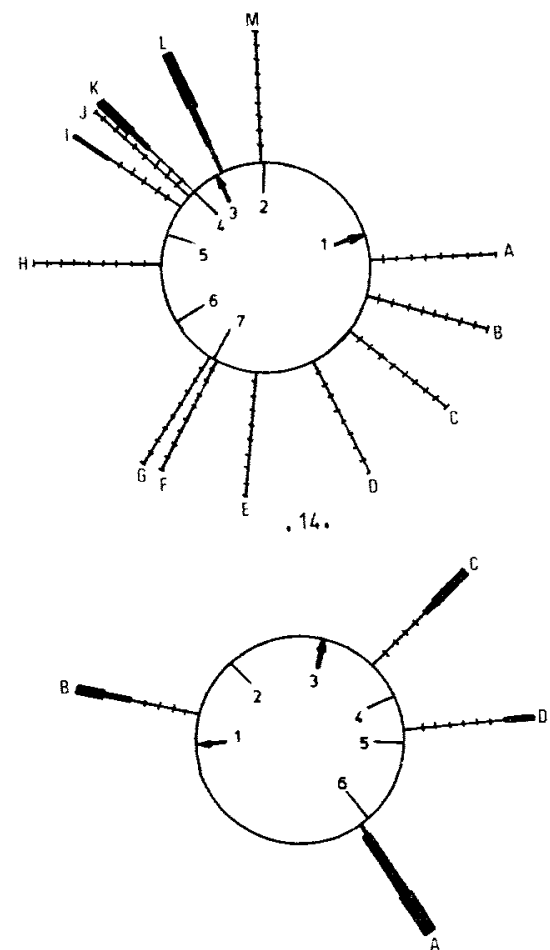

. 15 .

FiG. 6

Cépées ayant subi le traitement numéro $V$, répartition des dégâts.

Stumps which have undergone the ireatment number $V$, damage distribution. 


\section{Discussion et conclusions}

Les résultals de cette expérience montrent qu'une partie seulement du système racinaire peut être en relation avec tous les boutons (cépées 10-13-15). Dans beaucoup de cas, on constate quil y a réaction partielle de la partie aérienne dans des zones bien définies (cépées 11, 12, 14, 7, 9). Il existe done souvent des relations vasculaires nettement privilégiées entre certaines racines et certains boutons.

Le circuit de la sève le plus privilégié est souvent le circuit le plus court (distance minimale entre racine et rejet). Toutefois. une relation vasculaire privilégiée entre une racine et une zone aérienne ne signifie pas liaison unique et bien fermée entre ces 2 pôles. Dautre part, la zone irriguée par une racine donnée n'est pas nécessairement d’un seul tenant. Quelquefois, par exemple, 2 boutons opposés subissent des dégâts à la suite de l'injection dans une racine, alors que les boutons situés entre eux ne ressentent rien. 11 est donc difficile, voyant la position du bouton sur la cépéc, de déterminer a priori quelle partie du système racinaire va l'alimenter

Ainsi nous ne pouvons pas, dans le cas du bouleau. séparer la surface de la cépée en secteurs indépendants. Nous constatons ici que les individus (rejets) issus d'une coupe de laillis gardent des relations trophiques entre eux. L'individualisation trophique des brins de taillis se fait peu, contrairement au chêne-liège (Destremeau. Roderbourci, 1968). Nos résultats coïncident avec les conclusions tirées par Riedacker, (1973) sur eucalyptus après la 4" exploitation et par LAssoubierf (1980) sur le bananier.

La technique présentée ici a nécessité une «notation» des dégâts qui ne peut se faire que sur une échelle peu étendue (compromis entre une échelle très précise dans laquelle la "notation" deviendrait tout à fait subjective et une échelle trop peu précise. ne permettant que des conclusions grossières). Une manière de noter plus objeclive eat été par exemple de doser le produit à larrivée dans le bouton épicormique. Notons que la quantité de phytocide injectée induit la nuance des observations. Même si la réaction d'un rejet n'est pas directement proportionnelle à la quantité de phythormone qu'il reçoit. l'observation de l'évolution des dégâts sur une assez. grande période permet d'évaluer l’importance de la liaison vasculaire racine-rejet.

La simplicité de cette méthode perme une expérimentation à plus grande échelle et la prise en compte d'autres facteurs pouvant influer sur l'individualité des rejets Citons notamment lâge total de la souche, ainsi que le nombre de coupes qu'elle a subi. RIEDACKER (1973) a montré l'importance de ce phénomène sur eucalyptus. Après de nombreuses coupes, le réscau valsculaire devient probablement très complexe, ce qui pourrait expliquer les repousses différentes selon les cépées du même traitement dans notre cas. 


\section{Remerciements}

Cette étude s'est dérouléc dans le cadre d'un stage de $3^{\circ}$ année de l'Ecole Nationale des Ingénicurs des Eaux et Forêts, avec la collaboration de M. P. Cordon. Nous tenons à remercier M. H. Frocho' pour ses conscils en matière de phytocides.

\section{Summary}

Study of the distribution of crude sap between young birch coppice shoots by marking with a phytocide

The distribution of the rising sap from roots to sprouts has been studied by marking with a phytocide. On 15 stumps of a 3 years old birch coppice, injections of phytocide in the roots and observations of the subsequent damage on shoots showed that the stumps could not be separated in trophically independent sectors.

\section{Accepté pour publication le 7 arril 1983.}

\section{Références bibliographiques}

Destremeau D.X., Roderbourg J., 1968. Répartition de la sève entre les rejets. Am. Rech. For. Maroc, tome II, rapport 1968-1969, 237-242.

FINK V.S., 1980. Anatomische Untersuchungen über das Vorkommen von Spross und - Wurzelanlagen in Stammbereich von Laub-und Nadelbaümen 1 - Proventive Anlagen. Allg. Forst - u.J. Ztg., 151 (9).

FINK V.S., 1980. Anatomische Untersuchungen ïber das Vorkommen von Spross und Wurzelanlagen im Stammbereich von Laub und - Nadelbaümen 2 - Adventive Anlagen. Allg. Forst - u.J. Ztg., 151, 10, 181-194.

Lassoudiere A., 1980. Comportement du bananier cultivar «Poyo» al second cycle. Fruits, 25, 2, 69-93.

Pagìs L., 1982. Etude méthodologique de l'effet du recépage sur le système racinaire du bouleau (Betula verracesa et Betula pubescens). Rapport de stage de $3^{*}$ anné de I'E.N.I.T.E.F. - Document $n^{\prime \prime} 82 / 39$ de l'I.N.R.A., 1982, 105 p.

Riedacker A., 1973. Les taillis d'eucalyptus au Maroc. Ann. Rech. For. Maroc, tome 13, 157-349. 